\title{
Familial Eosinophilic Granulomatosis with Polyangiitis
}

\author{
Abdurhman S. Al Arfaj1 ${ }^{*}$, Mohammad Al Anazi², Najma Khalili', Akbar Ali Khan Pathan',3, \\ Narsimha Reddy Parine ${ }^{2}$
}

\author{
${ }^{1}$ Department of Medicine, Rheumatology Division, College of Medicine, King Saud University, Riyadh, KSA \\ ${ }^{2}$ Department of Biochemistry, College of Science, King Saud University, Riyadh, KSA \\ ${ }^{3}$ Integrated Gulf Biosystems, Riyadh, KSA \\ Email: *asarfaj@ksu.edu.sa
}

How to cite this paper: Al Arfaj, A.S., Al Anazi, M., Khalil, N., Pathan, A.A.K. and Parine, N.R. (2017) Familial Eosinophilic Granulomatosis with Polyangiitis. Open Journal of Rheumatology and Autoimmune Diseases, 7, 137-146.

https://doi.org/10.4236/ojra.2017.73013

Received: May 25, 2017

Accepted: July 29, 2017

Published: August 1, 2017

Copyright () 2017 by authors and Scientific Research Publishing Inc. This work is licensed under the Creative Commons Attribution International License (CC BY 4.0).

http://creativecommons.org/licenses/by/4.0/

\begin{abstract}
Eosinophilic granulomatosis with polyangiitis (EGPA) is an uncommon ANCA associated vasculitic disorder characterized by systemic necrotizing vasculitis of small vessels occurring exclusively among patients with bronchial asthma and tissue eosinophilia. Familial EGPA is extremely rare. Only two case reports have been published so far. We present a Saudi family with 3 cases of EGPA and almost three-fourths of family members affected by asthma. We explored genetic basis of EGPA in this family and found that genes were mutated in four affected siblings suggesting genetic involvement in susceptibility to EGPA.
\end{abstract}

\section{Keywords}

Familial Eosinophilic Granulomatosis with Polyangiitis, Churg-Strauss Syndrome, ANCA Associated Vasculitis

\section{Introduction}

Eosinophilic Granulomatosis with Polyangiitis (EGPA) formerly known as Churg-Strauss syndrome (CSS) is a vasculitic syndrome characterized by systemic necrotizing vasculitis of small vessels with extravascular granulomas occurring among patients with asthma and tissue eosinophilia and is included in spectrum of ANCA associated vasculitis (AAV) disorders [1] [2] [3]. Peripheral neuropathy is the chief neurological complication affecting almost 70\% of EGPA patients [4] [5]. It is a rare disease occurring with a prevalence of 10.7 to 14 per 1 million adults [6]. Familial EGPA cases are extremely rare and the only two articles found in literature comprised of a small number of cases [7] [8].

We herein report on a large family with EGPA and asthma affecting many 
members and probable genetic link of the disease. The family comprises of 15 members including father, mother, 5 sons and 8 daughters. Mother, 2 sons and 4 daughters are asthmatic. One son and 2 daughters have asthma and EGPA. Father, 2 sons and 2 daughters are normal. One of the sons, died during an exceptionally severe asthma attack. On the whole 10 members of the family have asthma and 3 of these asthmatic patients have EGPA.

\section{Observation}

\subsection{Asthma and EGPA Cases: (Pedigree-Figure 1)}

Case 1 (Sibling 3):

A 50-year-old Saudi female known asthmatic for 20 years presented to our hospital in November 2012 with gradual onset of right lower limb weakness which later on involved both the lower limbs and lead to inability to walk. There was no history of joint pain, no rash and no oral ulcers. She also had urinary incontinence. Physical examination of lower limbs showed normal bulk, increased tone, and power of $1 / 5$ in proximal and 3/5 in distal muscles, decreased reflex and upgoing plantars. Sensory and motor examination of upper and lower limbs was normal. CVS, respiratory and gastrointestinal examination were normal. Lab investigations showed hemoglobin $11 \mathrm{~g} / \mathrm{dl}$ (normal 12 - $16 \mathrm{gm} / \mathrm{dL}$ ), WBC count $4.2 \times 10^{9} / \mathrm{L}$ (normal $4-11 \times 10^{9} / \mathrm{L}$ ), eosinophils $7.4 \%$ (normal $0 \%-6 \%$ ), platelets $219 \times 10^{9} / \mathrm{L}$ (normal $140-450 \times 10^{9} / \mathrm{L}$ ), ESR $77 \mathrm{~mm} / \mathrm{hr}$ (elevated), CRP $172 \mathrm{mg} / \mathrm{L}$ (normal $1-6 \mathrm{mg} / \mathrm{L}$ ), normal urea, serum creatinine, and liver function tests. Hepatitis B and C serology were negative. ANA, anti DNA, ANCA, pANCA and c-ANCA were all negative. Nasal swab was normal. Diagnosis pictures of her chest $\mathrm{X}$ ray showed prominent left hilar shadow and both lung fields clear and CT lung showed bilateral basal focal consolidation. MRI spine showed spinal cord swelling extending from C3-T1 with enhancement and long segment cervical cord high signal intensity on T2 suggestive of transverse myelitis. Computed tomography (CT) scan of chest, abdomen and pelvis were unremarkable. CSF analysis revealed normal cell count and differentials, normal protein and glucose. Nerve conduction study showed normal sensory studies, with positive axonal motor neuropathy. She was diagnosed as a case of EGPA with transverse myelitis. Upon admission she was started on methyl prednisolone IV OD for 5 days. She was then maintained on oral prednisolone $1 \mathrm{mg} / \mathrm{kg}$ OD. She received IV cyclophosphamide 500 - $700 \mathrm{mg}$ monthly for 6 doses. She received prednisolone $20 \mathrm{mg}$ PO OD which was finally tapered to $5 \mathrm{mg}$ PO OD, pregabalin $75 \mathrm{mg}$ PO bid and physiotherapy. She improved after 1 month of treatment. After completion of 6 doses of IV cyclophosphamide she was shifted to azathioprine $50 \mathrm{mg}$ PO bid and also received enoxaparin $40 \mathrm{mg}$ PO OD. In July 2013 she was referred to a specialized rehabilitation center for further physiotherapy and in May 2014 she improved, was on physiotherapy and walking with aid. During her last follow up in August 2016 she was doing well.

Case 2 (Sibling 7)

A 39-year-old female, known asthmatic since childhood, presented to our 
hospital in February 2012 with history of recurrent upper abdominal pain for the past one year, colicky in nature, radiating to back, increased by food intake. Vomiting was projectile with blood, loss of appetite, diarrhea with fresh blood. She had history of intermittent melena for one month. She had small joints pain but no skin rash or oral ulcer. She later developed left lower limb numbness. Physical examination showed no active synovitis, no visceromegaly or tenderness in epigastrium and periumbilical region. Examination of lower limbs revealed absent ankle jerk and decreased sensation in left lower limb. Lab investigations showed hemoglobin $9.4 \mathrm{~g} / \mathrm{dl}$, WBC count $15 \times 10^{9} / \mathrm{L}$, eosinophils $49 \%$, eosinophils absolute count was $735 / \mu \mathrm{L}$ (normal $<350 / \mu \mathrm{L}$ ), platelets $213 \times 10^{9} / \mathrm{L}$, normal urea and serum creatinine, alkaline phosphatase 320 IU/L (normal 39 - 117 IU/L), aspartate aminotransferase 72 IU/L (normal 10 - $31 \mathrm{IU} / \mathrm{L}$ ) and alanine aminotransferase 114 IU/L (normal 10 - 31 IU/L) and urine protein 1+. Hepatitis B and C serology were negative. ANA, anti DNA, ANCA, p-ANCA and $c$-ANCA were all negative. Nerve conduction study showed leg mixed motor and sensory nerves worse on left side, axonal polyneuropathy in both legs. Diagnosis by ultrasound scan of abdomen was normal, upper GI endoscopy revealed incompetent cardia, erosive gastritis and erosive duodenitis, capsule endoscopy showed multiple duodenal and jejunal ulcers, endoscopic histology showed chronic gastritis and chronic duodenitis. CT scan of abdomen and colonoscopy were normal. CT angiography showed no evidence of intestinal ischemia. Nerve conduction study showed axonal polyneuropathy in both legs. She was diagnosed with EGPA. During her first admission in July 2012 she was started on methyl prednisolone $40 \mathrm{mg}$ IV TID. Later she was started on IV cyclophosphamide monthly. She received 6 doses of 500 - $600 \mathrm{mg}$ of IV cyclophosphamide monthly from July 2012 until Dec 2012. She was also treated with oral prednisolone $70 \mathrm{mg}$ PO OD which was tapered to $5 \mathrm{mg}$ PO OD in Nov 2013. After completion of IV cyclophosphamide doses she was shifted to azathioprine $75 \mathrm{mg}$ OD and gabapentin $40 \mathrm{mg}$ PO bid. During her last follow up in Nov 2013 she was asymptomatic apart from numbness of left leg and soles. She was doing well on oral prednisolone $5 \mathrm{mg}$ PO OD and azathioprine $75 \mathrm{mg}$ OD.

Case 3 (Sibling 9)

A 34-year-old male, known to have bronchial asthma since 2006 presented to our hospital in October 2010 with upper abdominal pain for last few weeks, intermittent in character increased by food intake, with history of on and off vomiting diarrhea with melena and weight loss of $5 \mathrm{~kg}$. He had history of right sided hemiparesis with complete recovery and myocarditis in 2006. There was history of rash involving the face and leg, pink in color and history of joint pain in the knee, wrist and ankle. On examination there was no visceromegaly or tenderness in epigastrium, respiratory system was normal and there was erythematous rash on the legs. CNS exam was normal. Lab investigations showed $\mathrm{Hb} 17.1 \mathrm{~g} / \mathrm{dl}$, WBC count $14.3 \times 10^{9} / \mathrm{L}$, eosinophils $58 \%$, eosinophil absolute count was $829 / \mu \mathrm{L}$, platelets $282 \times 10^{9} / \mathrm{L}$, ESR $27 \mathrm{~mm} / \mathrm{hr}$ (elevated), CRP 184 $\mathrm{mg} / \mathrm{L}$, urea $7.3 \mathrm{mmol} / \mathrm{L}$ (normal 2.5 - $6.4 \mathrm{mmol} / \mathrm{L}$ ), serum creatinine 118 $\mathrm{mmol} / \mathrm{L}$ ( normal $53-106 \mathrm{mmol} / \mathrm{L}$ ) and urine protein $1+$. Hepatitis B and C se- 
rology, ANA, anti DNA, ANCA, ANCA-MPO and ANCA-PR3 were all negative. Diagnosis picture by ultrasound scan of abdomen showed mildly enlarged liver with thickened walls of gall bladder, slightly high echogenecity was seen in both kidneys, Upper GI endoscopy showed normal esophagus with gastritis, and duodenitis. Lower GI endoscopy showed caecal congestion and rectosigmoid erythema. Gastric biopsy showed mild chronic gastritis and biopsy of caecum and colon were unremarkable. PFT was normal. Echochardiogrpahy showed small pericardial effusion. CT chest showed features of mild bronchial asthma with ill-defined ground glass appearance suggestive of vasculitis. MRI brain showed multiple high signal intensity in deep white matter of left cerebral hemisphere, most likely vasculopathy. CT abdomen showed patent mesenteric vessels slightly congested, minimal free pelvic fluid and CT angiography showed no evidence of ischemia. He was diagnosed with EGPA. During admission in Oct 2010 patient was started on methyl prednisolone $40 \mathrm{mg}$ IV TID, later decreased to $10 \mathrm{mg}$ IV TID. Patient was started on IV cyclophosphamide 700 - 800 mg monthly for 6 doses from Nov 2010 until May 2011. Oral prednisolone was started with a dose of $40 \mathrm{mg} \mathrm{OD}$, tapered to $5 \mathrm{mg} \mathrm{OD}$. He improved and was later shifted to azathioprine $50 \mathrm{mg}$ OD which was increased to $100 \mathrm{mg}$ OD. He was later admitted in April 2012 with shortness of breath, cough, wheezing and treated with methyl prednisolone $80 \mathrm{mg} \mathrm{q} 8 \mathrm{~h}$, azathioprine $75 \mathrm{mg}$ OD, oral prednisolone $5 \mathrm{mg}$ OD. During his last follow up in April 2016 he was asymptomatic on oral prednisolone tapered to $2.5 \mathrm{mg}$ OD, and azathioprine $75 \mathrm{mg}$ OD.

\subsection{Asthma Cases: (Pedigree-Figure 1)}

Sibling 2: A 53-year-old male, asthmatic on Ventolin.

Sibling 4: A 48-year-old female, asthmatic on Ventolin.

Sibling 6: A 41-year-old female, asthmatic on Ventolin. She has lower limb numbness for more than 2 years.

Sibling 8: A 37-year-old female, asthmatic for 7 years, on oral prednisolone and daily seretide at bedtime. She had pleural effusion, pericardial effusion; pleural tap was done and responded to prednisolone.

Sibling 10: A 33-year-old female, asthmatic for 4 years on Ventolin. She had history of left sided pleural effusion treated with oral prednisolone $40 \mathrm{mg}$ OD for

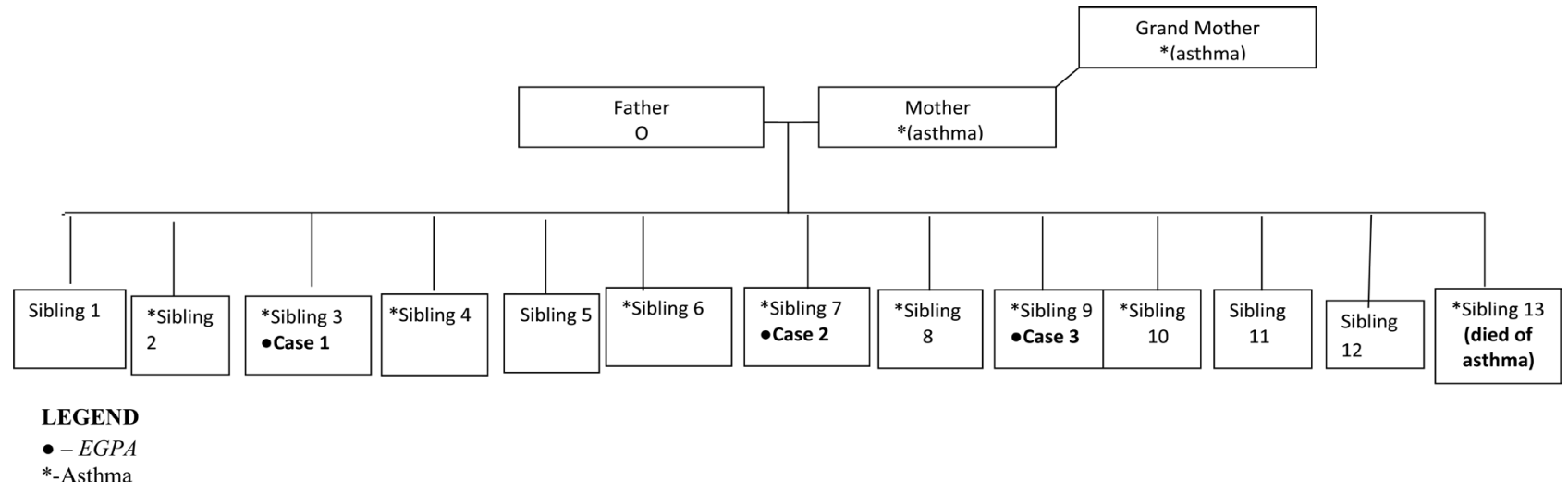

Figure 1. Family pedigree. 
1 month and history of maculopapular rash on face, elbow, posterior aspect of knee and multiple scaly plaques on scalp. Lab investigations showed mild proteinuria, ANCA, ANCA-MPO and ANCA-PR3 were negative. She was treated with oral prednisolone $5 \mathrm{mg}$ OD.

Sibling 13: A 30-year-old male, died of severe asthmatic attack few years back. He was followed up elsewhere.

\subsection{Methods}

Informed consent was obtained from the patients to perform the study. To explore if genetics played a role in such high number of cases in a family, we performed whole exome sequencing of four affected individuals along with two unaffected. We captured and sequenced coding regions to a mean coverage of $100 \times$ and sufficient depth to call variants at $\sim 97 \%$ of each targeted exome, filtering against public dbSNP and $1000 \mathrm{G}$ databases.

\section{Exome sequencing}

Total DNA was extracted using QIAamp DNA Blood Mini Kit (Qiagen). Extracted DNA was quantified using a Qubit Fluorimeter (Life Technologies). Libraries for sequencing were prepared according to the TruSeq DNA Sample Preparation Kit EUC 15026489 (Illumina). Approximately, 1 - 2 ug of DNA was fragmented according to SureSelect Protocol. After fragmentation, all samples were subjected to end-repair, A-tailing, and adaptor ligation of Illumina Multiplexing PE adaptors. The adapter-ligated fragments were enriched subsequently by PCR followed by Agencourt AMPure Beads (Beckman Coulter) purification. To capture the whole Exome pre-pooling with equimolar amounts was performed and enriched in 5- or 6-plex reactions as per the TruSeq Exome Enrichment Kit Protocol (EUC 15013230). The built libraries were checked on a Bioanalyzer using High Sensitivity DNA chip (Agilent Technologies), while library concentrations were measured by quantitative PCR. Clustering of the pooled DNA were performed on cBot instrument (Illumina) using the TruSeq PE Cluster Kit. Real time analysis (RTA) for base calling was performed with RTA (1.12.4.2 or 1.13.48) and the resulting BCL files were converted to FASTQ format using CASAVA 1.7 or 1.8 (Illumina) after filtering and de-multiplying. Sample sequences were aligned to the human reference genome hg19GRCh37 using BWA [9], PCR duplicates were removed with Picard. The SNPs and Indels are detected by SAMTOOLS (http://samtools.sourceforge.net/) and compared to the dbSNP \& $1000 \mathrm{G}$ variant Database.

\section{Mutation annotation}

The captured mutations in variant call format were further annotated by ANNOVAR [10] in an excel file with the complete dbSNP identity, amino acid change and gene location information [11], allele frequencies were calculated using 1000 genome project information [12], and functional prediction were carried out using SIFT [13] and Polyphen2 [14].

Confirmation studies by Sanger sequencing

The PCR primers were designed using Primer3web [15] for all the 15 immune 
dysfunction genes and were amplified in all the familial samples. PCR products were purified using BigDye terminator (Applied Biosystems) and sequenced using BigDye terminator ver 3.1 (Applied Biosystems) and analyzed on an Abi 3130 genetic analyzer. The sequences obtained were visualized and analyzed using FinchTV [16] and CodonCode Aligner [17].

\subsection{Results}

\section{Exome sequencing}

We identified non-synonymous single nucleotide variants, which were commonly expressed among 4 affected members of this family and absent in the healthy individuals. These variants were found in 103 different genes involved in different metabolic pathways; Table 1 . Interestingly 15 genes related to immune dysfunction were specially mutated in the 4 affected individuals of which NAV2 which encodes a member of the neuron navigator gene family, and SFTPC encodes the pulmonary-associated surfactant protein $\mathrm{C}$ were directly related to asthma (positive disease association) (Table 1).

Sanger sequencing

Sequencing results from the 44 genes tested (data not shown) in all the familial samples showed a strong relationship between the complex inheritances of this disease among the family members. Further the role of Eosinophilic granulomatosis with polyangiitis is an HLA-associated disease was also explored by sequencing all the promoter region and 5 exons of the human IL-10 gene.

\section{Discussion}

We present a family of fifteen members of whom 10 members have asthma, 3 siblings have fully confirmed EGPA and one sibling died from severe asthmatic attack. Siblings 8 and 10 have asthma, skin rashes, pleural and pericardial effusion responding to prednisolone, most likely they have the same diagnosis of EGPA. Familial association of EGPA is very rare and to our knowledge only two studies have reported familial cases of definite EGPA so far [7] [8] and one study reported familial cases of a definite and a probable EGPA [18]. The first report of familial EGPA was described in 2007 in 2 asthmatic sisters [7]. The study investigated the human leukocyte antigen (HLA) typing of the two sisters and their six living siblings to elucidate the heritability of EGPA. HLA-A, B, DRB1, DQA1, DQB1, DPB1 were analyzed, but found no significant evidence of association of HLA haplotypes with susceptibility to EGPA.

A study in 2003 reported the familial clustering of EGPA and granulomatosis with polyangiitis (GPA) formerly known as Wegener's granulomatosis (WG) in a father and his son respectively [19]. Both father and son lived together in urban area and the study found that both shared the HLA haplotype $A * 03 ; B * 07$; $C *$ W07; DRB1 * 0404; DQB1 * 0302. The study suggested the pathogenic role of genetic factors in familial clustering of vasculitic diseases. Familial clustering of different systemic vasculitides had been previously described with simultaneous or later occurrence in the same family [19] [20] [21] [22]. The most com- 
Table 1. Exome sequencing in 15 patients.

\begin{tabular}{|c|c|c|c|}
\hline Gene & SNP & Phenotype & Disease Class \\
\hline HSPG2 & rs1138469 & periodontitis & IMMUNE \\
\hline SFTPC & rs 4715 & asthma respiratory syncytial virus & IMMUNE \\
\hline TMEM132D & rs140762080 & Celiac Disease| & IMMUNE \\
\hline SPEF2 & rs 16902381 & Celiac Disease| & IMMUNE \\
\hline FAM129A & rs35601690 & Celiac Disease| & IMMUNE \\
\hline NGF & rs6330 & Multiple Sclerosis, Dermatitis, Atopic & IMMUNE \\
\hline TNIP1 & rs52817781 & Psoriasis, Lupus Erythematosus, Systemic & IMMUNE \\
\hline IKBKE & rs17021877 & Arthritis, Rheumatoid| & IMMUNE \\
\hline MAPKAPK2 & rs55894011 & Arthritis, Rheumatoid| & IMMUNE \\
\hline NAV2 & rs 12421330 & Asthma & IMMUNE \\
\hline NAV2 & rs3736101 & Asthma & IMMUNE \\
\hline NAV2 & rs3729989 & Asthma & IMMUNE \\
\hline NAV2 & rs11229301 & Asthma & IMMUNE \\
\hline NAV2 & rs55944888 & Asthma & IMMUNE \\
\hline NAV2 & rs 17686210 & Asthma & IMMUNE \\
\hline NAV2 & rs11229281 & Asthma & IMMUNE \\
\hline NAV2 & rs11606506 & Asthma & IMMUNE \\
\hline NAV2 & rs7130086 & Asthma & IMMUNE \\
\hline NAV2 & rs139333041 & Asthma & IMMUNE \\
\hline NAV2 & rs200336178 & Asthma & IMMUNE \\
\hline NAV2 & rs 17150411 & Asthma & IMMUNE \\
\hline NAV2 & rs2297781 & Asthma & IMMUNE \\
\hline NAV2 & rs199946104 & Asthma & IMMUNE \\
\hline TEC & rs17013181 & Inflammatory Bowel Diseases & IMMUNE \\
\hline TEC & rs28403003 & Inflammatory Bowel Diseases & IMMUNE \\
\hline TNP2 & rs 16957558 & Diabetes Mellitus, Type 1 & IMMUNE \\
\hline TNP2 & rs2290182 & Diabetes Mellitus, Type 1 & IMMUNE \\
\hline TNP2 & rs78847227 & Diabetes Mellitus, Type 1 & IMMUNE \\
\hline TNP2 & rs144244672 & Diabetes Mellitus, Type 1 & IMMUNE \\
\hline BLK & rs 4715 & Arthritis, Rheumatoid, Lupus Erythematosus, Systemic & IMMUNE \\
\hline BLK & rs148981308 & Arthritis, Rheumatoid, Lupus Erythematosus, Systemic & IMMUNE \\
\hline BLK & rs4731 & Lupus Erythematosus, Systemic & IMMUNE \\
\hline BLK & rs144215650 & Lupus Erythematosus, Systemic & IMMUNE \\
\hline REV3L & rs458017 & Psoriasis & IMMUNE \\
\hline FCRL3 & rs79889663 & Graves Disease, Diabetes Mellitus, Type 1 & IMMUNE \\
\hline FCRL3 & rs3795331 & Diabetes Mellitus, Type 1 & IMMUNE \\
\hline
\end{tabular}


mon type of familial occurrence of GPA was among siblings [22].

The cause of EGPA is unknown but is probably multifactorial. The familial association of EGPA suggests that genetic factors may confer susceptibility to EGPA [23]. However, HLA typing studies did not yield conclusive results [7]. The hypothesis that environmental agents may trigger the disease in genetically predisposed subjects implies variations in genes encoding for proteins crucial for the immune response regulation [24].

We explored the genetic basis of EGPA in this large family of multiple cases of EGPA. Exome sequencing of a small number of affected individuals is a powerful, efficient strategy for identifying the genes underlying rare Mendelian disorders and will likely transform the genetic analysis of monogenic traits. In the present study we have a family of Saudi Arabian origin where 9 out of 15 individuals were suffering from different forms of EGPA.

We found several genes mutated in the four affected siblings suggesting genetic involvement in susceptibility to EGPA. The only review article which presents the association of genetic determinants in EPGA with HLA is by Andrea Gioffredi (2014), where the author cited many references which prove the association of HLA-DRB1 ${ }^{\star} 04,{ }^{*} 07$ and HLA-DRB4 with EPGA [25]. Many SNP's of the IL10 gene, an important molecule for the activation of Th-2 pathway were shown associated with EGPA pathogenesis. Similar results were obtained in our present study where we identified (317) G > GC, (272) C > CT in the promotor region of two of the siblings affected with severe asthma. Surprisingly all the affected individuals showed a (179) A > AG mutation in the exon 5, which is being researched further in detail.

\section{Conclusion}

Based on the observations we propose for the first time that there is a clear role of genetics in Eosinophilic Granulomatosis with Polyangiitis and this familial sample could be the best example of a complex inheritance.

\section{References}

[1] Churg, J. and Strauss, L. (1951) Allergic Granulomatosis, Allergic Angiitis, and Periarteritis Nodosa. The American Journal of Pathology, 27, 277-301.

[2] Jennette, J.C., Falk, R.J., Andrassy, K., Bacon, P.A., Churg, J., Gross, W.L., et al. (1994) Nomenclature of Systemic Vasculitides. Proposal of an International Consensus Conference. Arthritis \& Rheumatology, 37, 87-92.

[3] Hoffman, G.S. and Pecks, U. (1998) Antineutrophil Cytoplasmic Antibodies. Arthritis \& Rheumatology, 41, 1521-1537. https://doi.org/10.1002/1529-0131(199809)41:9<1521::AID-ART2>3.0.CO;2-A

[4] Sehgal, M., Swanson, J.W., DeRemee, R.A. and Colby, T.V. (1995) Neurologic Manifestations of Churg-Strauss Syndrome. Mayo Clinic Proceedings, 70, 337-341. https://doi.org/10.4065/70.4.337

[5] Keogh, K.A. and Specks, U. (2003) Churg-Strauss Syndrome: Clinical Presentation, Antineutrophil Cytoplasmic Antibodies, and Leukotriene Receptor Antagonists. The American Journal of Medicine, 115, 284-290. 
https://doi.org/10.1016/S0002-9343(03)00359-0

[6] Mahr, A., Guillevin, L., Poissonnet, M. and Aymé, S. (2004) Prevalences of Polyarteritis Nodosa, Microscopic Polyangiitis, Wegener's Granulomatosis, and ChurgStrauss Syndrome in a French Urban Multiethnic Population in 2000: A Capture-Recapture Estimate. Arthritis \& Rheumatology, 51, 92-99. https://doi.org/10.1002/art.20077

[7] Tsurikisawa, N., Morita, S., Tsuburai, T., Oshikata, C., Ono, E., Taniguchi, M., Saito, H., Yanagihara, Y. and Akiyama, K. (2007) Familial Churg-Strauss Syndrome in Two Sisters. Chest, 131, 592-594. https://doi.org/10.1378/chest.06-1208

[8] Alyasin, S., Khoshkhui, M. and Amin, R. (2015) Familial Churg-Strauss Syndrome in a Sister and Brother. Iranian Journal of Allergy, Asthma and Immunology, 14, 338-340.

[9] Li, H. and Durbin, R. (2009) Fast and Accurate Short Read Alignment with Burrows-Wheeler Transform. Bioinformatics, 25, 1754-1760. https://doi.org/10.1093/bioinformatics/btp324

[10] Wang, K., Li, M. and Hakonarson, H. (2010) ANNOVAR: Functional Annotation of Genetic Variants from High-Throughput Sequencing Data. Nucleic Acids Research, 38, e164. https://doi.org/10.1093/nar/gkq603

[11] Sherry, S.T., Ward, M.H., Kholodov, M., Baker, J., Phan, L., Smigielski, E.M., et al. (2001) dbSNP: The NCBI Database of Genetic Variation. Nucleic Acids Research, 29, 308-311. https://doi.org/10.1093/nar/29.1.308

[12] Altshuler, D.M., Durbin, R.M., Abecasis, G.R., Bentley, D.R., Chakravarti, A., Clark, A.G., et al. (2012) An Integrated Map of Genetic Variation from 1,092 Human Genomes. Nature, 491, 56-65. https://doi.org/10.1038/nature11632

[13] Kumar, P., Henikoff, S. and Ng, P.C. (2009) Predicting the Effects of Coding NonSynonymous Variants on Protein Function Using the SIFT Algorithm. Nature Protocols, 4, 1073-1081. https://doi.org/10.1038/nprot.2009.86

[14] Adzhubei, I.A., Schmidt, S., Peshkin, L., Ramensky, V.E., Gerasimova, A., Bork, P., et al. (2010) A Method and Server for Predicting Damaging Missense Mutations. Nature Methods, 7, 248-249. https://doi.org/10.1038/nmeth0410-248

[15] Untergasser, A., Cutcutache, I., Koressaar, T., Ye, J., Faircloth, B.C., Remm, M., et al. (2012) Primer3-New Capabilities and Interfaces. Nucleic Acids Research, 40, e115. https://doi.org/10.1093/nar/gks596

[16] FinchTV V1.4. (2006) A Brilliant Trace Viewer. Geospiza, Inc. http://www.geospiza.com/Products/finchtv.shtml

[17] Rozen, S. and Skaletsky, H. (2000) Primer3 on the WWW for General Users and for Biologist Programmers. Methods in Molecular Biology, 132, 365-386.

[18] Ota, M., Takenaka, K., Takahashi, M. and Nagasaka, K. (2012) Eosinophilia with Organ Involvement in 3 Siblings. Japanese Journal of Clinical Immunology, 35, 533-538. https://doi.org/10.2177/jsci.35.533

[19] Manganelli, P., Giacosa, R., Fietta, P., Zanetti, A. and Neri, T.M. (2003) Familial Vasculitides: Churg-Strauss Syndrome and Wegener's Granulomatosis in 2 FirstDegree Relatives. The Journal of Rheumatology, 30, 618-621.

[20] Fietta, P., Manganelli, P., Zanetti, A. and Neri, T.M. (2002) Familial Giant Cell Arteritis and Polymyalgia Rheumatica: Aggregation in 2 Families. The Journal of Rheumatology, 29, 1551-1555.

[21] Rottem, M., Cotch, M.F., Fauci, A.S. and Hoffman, G.S. (1994) Familial Vasculitis: Report of 2 Families. The Journal of Rheumatology, 21, 561-563. 
[22] Hay, E.M., Beaman, M., Ralston, A.J., Ackrill, P., Bernstein, R.M. and Holt, P.J. (1991) Wegener's Granulomatosis Occurring in Siblings. British Journal of Rheumatology, 30, 144-145. https://doi.org/10.1093/rheumatology/30.2.144

[23] Griffith, M.E. and Pusey, C.D. (1997) HLA Genes in ANCA-Associated Vasculitides. Experimental and Clinical Immunogenetics, 14, 196-205.

[24] Huang, D., Zhou, Y. and Hoffman, G.S. (2001) Pathogenesis: Immunogenetic Factors. Best Practice \& Research: Clinical Rheumatology, 15, 239-258. https://doi.org/10.1053/berh.2001.0142

[25] Gioffredi, A., Maritati, F., Oliva, E. and Buzio, C. (2014) Eosinophilic Granulomatosis with Polyangiitis: An Overview. Frontiers in Immunology, 5, 549.

https://doi.org/10.3389/fimmu.2014.00549

\section{Scientific Research Publishing}

\section{Submit or recommend next manuscript to SCIRP and we will provide best} service for you:

Accepting pre-submission inquiries through Email, Facebook, LinkedIn, Twitter, etc. A wide selection of journals (inclusive of 9 subjects, more than 200 journals)

Providing 24-hour high-quality service

User-friendly online submission system

Fair and swift peer-review system

Efficient typesetting and proofreading procedure

Display of the result of downloads and visits, as well as the number of cited articles

Maximum dissemination of your research work

Submit your manuscript at: http://papersubmission.scirp.org/

Or contact ojra@scirp.org 